\title{
Adaptive control for car like vehicles guidance relying on RTK GPS: rejection of sliding effects in agricultural applications
}

\author{
R. Lenain ${ }^{\star}$, B. Thuilot ${ }^{\diamond}$ C. Cariou ${ }^{\star}$, P. Martinet ${ }^{\diamond}$ \\ * Cemagref \\ $\diamond$ LASMEA \\ BP 50085 - 24, av. des Landais \\ 63172 Aubi ere Cedex F rance \\ 24, av. des Landais \\ roland.lenain@cemagref.fr \\ 63177 Aubi ere Cedex F rance \\ Benoit.Thuilot@lasmea.univ-bpclermont.fr
}

\begin{abstract}
A bstract- Numerous agricultural applications require very accurate guidance of farm vehicles. Current w orks ha e established that RTK GPS was a very suitable sensor in order to meet the expected precision: sev eral control laws ha vebeen designed for $v$ ehicles equipped with such a sensor, and satisfactory results hav e been achieved as long as vehicles do not slide. Nevertheless, in actual $\mathrm{w}$ orking conditions (sloping fields, entering into curves on a wet land, ...), sliding inevitably occurs. In this paper, w edesign a nonlinear adaptive control la $\mathrm{w}$ in order to preserve guidance precision in presence of sliding: realtime sliding estimation is used to correct v ehicle ev olution. Field experiments, demonstrating the capabilities of that control scheme are reported and discussed.
\end{abstract}

Keywords: mobile robots, nonlinear control systems, adaptive control la ws, kinematic GPS, agriculture.

\section{INTRODUCTION}

Many researc h groups and manufacturers are currently $\mathrm{w}$ orking on rehicles automatic guidance, devoted to agricultural tasks. The main objective is to increase the driving accuracy. $\mathrm{P}$ oten tial applications are for instance achieving perfectly parallel runs when seeding, harv esting, ro w cropping, ... or eliminating skips and/or ov erlaps when spraying fertilizers or pesticides, ... In addition, automatic guidance delivers the human operator from the tiring driving task. He can then fully dev otehis time to the tools monitoring. Quality of the agronomic work carried out and productivity can clearly be improved.

Since RTK GPS (also named kinematic GPS) can provide realtime absolute position with a centimeter accuracy, it is often used as the keystone of the perception sensing system in these automatic guided vehicles. This sensor is here fully reliable, since in terruptions in GPS signal reception, which is one major concern, do not occur in agricultural tasks where vehicles move on open fields. V arious systems relying upon a GPS antenna and additional inertial sensors (e.g. [7]), on multiple GPS antennas (e.g. [8]), or upon a unique GPS antenna (e.g. [11]) have been inv es- tigated. Some guidance devices ha ve already beenmarketed: e.g. the BEELINE Navigator (relying on 1 GPS antenna and an Inertial Navigation System (INS)) and the $A$ utoFirm System (making use of 3 GPS antennas) ha ve been inroduced from the late nineties, respectively by the Australian company A gSystemsand the American company Inte griNautics GPS systems suppliers as well as agricultural manufacturers are also investing in this market: T rimble is selling the AgGPS A utopilotwhen manufacturer John De ereproposes a completely automated tractor, using several sensors (GPS, vision, NIR) [9].

These devices are mainly dev otedto applications where the $\mathrm{v}$ ehicle mist execute perfectly straight lines, and make use ofsev eral sensors. Current dev elopmerts aim at ex tending the performances of the automatic guided vehicles and simultaneously reducing their cost. In previous w orks(see [11] and [2]), w eha veaddressed this twofold challenge. On one hand, curved path following cap ability has been addressed. A nonlinear control la w, taking into account for the reference path curvature, has been designed. Automatic half-turns and field boundaries following have been successfully experimented, see [11]. On the other hand, our sensing device consists in a unique kinematic GPS. Multiple sensors are efficient, since they provide control designer with the whole vehicle attitude, but are quitexpensiv e. In [11] and [2], the whole state vector is deriv edfrom the information pro vided by the unique GPS antenna via a Kalman state reconstructor.

Satisfactory experiments reported in [11] have been conducted on a dry and ev en ground. How ev er,it has also been observed that the guidance accuracy is slightly reduced when the vehicle enters into a curve on a wet land, or when the vehicle mov es on sloping fields. In both cases, the trac king error originates from the occurrence of sliding. The aim of this paper is to present further dev elopments of the guidance algorithm described in [11], in order to reject sliding effe cts. The paper is organized as follows: farm tractor modelling and control under nonsliding assumption is first recalled in section II. Next, 
sliding modelling is discussed in section III. The design of the adaptive control law, which allows to reject sliding effects, is then detailed in section IV. Finally, experimental results are displayed in section $\mathrm{V}$.

\section{VEHICLE GUIDANCE UNDER NON-SLIPPING ASSUMPTION}

\section{A Vehicle modelling}

Vehicle modelling relies upon celebrated Ackermann's model, also named bicycle model. More precisely, the two front wheels and the two rear wheels are considered equivalent as unique virtual wheels located at mid-distance between respectively actual front wheels and rear wheels, see Figure 1. These assumptions are quite common in Automatic literature, see for instance [13].

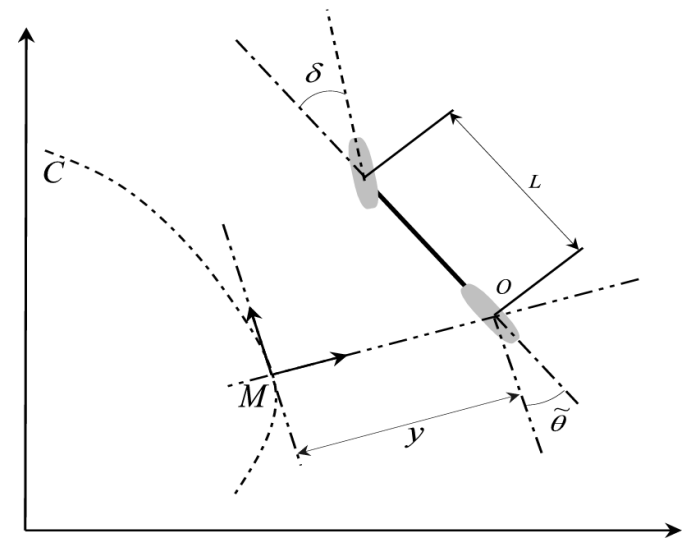

Figure 1: Vehicle modelling parameters

Our notations are detailed hereafter (see also Figure 1):

- $\mathcal{C}$ is the path to be followed,

- $O$ is the center of vehicle virtual rear wheel,

- $M$ is the point on $\mathcal{C}$ which is the closest to $O$. $M$ is assumed to be unique, which is realistic when the vehicle remains quite close from $\mathcal{C}$.

- $s$ is the curvilinear coordinate of point $M$ along $\mathcal{C}$, and $c(s)$ denotes the curvature of $\mathcal{C}$ at that point.

- $y$ and $\tilde{\theta}$ are respectively lateral and angular deviation of the vehicle with respect to reference path $\mathcal{C}$ (see Figure 1).

- $\delta$ is the virtual front wheel steering angle.

- $v$ is the vehicle linear velocity, considered here as a parameter, whose value may be time-varying during the vehicle evolution.

- $L$ is the vehicle wheelbase.
Vehicle configuration can be described without ambiguity by the state vector $(s, y, \tilde{\theta})$ : the two first variables provide point $O$ location, and the last one describes the vehicle heading. Since $v$ is considered as a parameter, the only vehicle control variable is $\delta$. It can then be established, see for instance [11], that under non-sliding assumption, vehicle kinematic state space model is:

$$
\left\{\begin{aligned}
\dot{s} & =\frac{v \cos \tilde{\theta}}{1-c(s) y} \\
\dot{y} & =v \sin \tilde{\theta} \\
\dot{\tilde{\theta}} & =v\left(\frac{\tan \delta}{L}-\frac{c(s) \cos \tilde{\theta}}{1-c(s) y}\right)
\end{aligned}\right.
$$

Model (1) is clearly singular when $y=\frac{1}{c(s)}$, i.e. when point $O$ is superposed with the path $\mathcal{C}$ curvature center at abscissa $s$. However, this configuration is never encountered in practical situations.

\section{$B$ Curved path following}

The objective of curved path following is to bring and keep $y$ and $\tilde{\theta}$ equal to 0 , independently from variable $s$ evolution (which mainly depends on the value of parameter $v$ ). The control approach proposed in [11] consists in pointing out that nonlinear model (1) can be converted, without any approximation, into linear equations. More precisely, reporting into model (1) the invertible nonlinear state transformation:

$$
\Theta((s, y, \tilde{\theta}))=\left(a_{1}, a_{2}, a_{3}\right) \triangleq(s, y,(1-c(s) y) \tan \tilde{\theta})
$$

and describing the vehicle evolution with respect to $s$ (instead of with respect to time) leads to the linear model:

$$
\left\{\begin{aligned}
\frac{d a_{2}}{d s} & =a_{3} \\
\frac{d a_{3}}{d s} & =m_{3}
\end{aligned}\right.
$$

Computations show that the new control variable $m_{3}$ is related in an invertible way with the actual one, i.e. $\delta$. Such a transformation can be achieved, since model (1) enters into the class of nonlinear systems which can be converted into chained form, see for instance [10].

Celebrated linear control theory can then be used to design control law $m_{3}$ in order to bring $\left(a_{2}, a_{3}\right)$ to 0 . In view of (2) $y$ and $\tilde{\theta}$ are also brought to 0 , as desired. The inversion of the nonlinear relation between $m_{3}$ and $\delta$ provides us with the actual nonlinear control law:

$$
\begin{aligned}
\delta(y, \tilde{\theta})= & \arctan \left(L \left[\frac { \operatorname { c o s } ^ { 3 } \tilde { \theta } } { ( 1 - c ( s ) y ) ^ { 2 } } \left(\frac{d c(s)}{d s} y \tan \tilde{\theta}\right.\right.\right. \\
& -K_{d}(1-c(s) y) \tan \tilde{\theta}-K_{p} y \\
& \left.\left.\left.+c(s)(1-c(s) y) \tan ^{2} \tilde{\theta}\right)+\frac{c(s) \cos \tilde{\theta}}{1-c(s) y}\right]\right)
\end{aligned}
$$

Closed-loop performances can be adjusted by tuning parameters $\left(K_{p}, K_{d}\right)$. The attractive feature of control law (4) is that nonlinear system (1) is controlled just as a linear one, thus taking advantage of Linear Systems Theory, without achieving any approximation. 


\section{SLIDING MODELLING}

A first possibility, in order to take into account for sliding phenomenon, is to consider dynamic models, see for instance [4], [12], ... However since such models describe all tractor features (inertia, slipping, springing,...), they are very large, and therefore not very tractable. Moreover, they encompass numerous parameters (masses, wheelground contact conditions, spring stiffness,...) whose values are badly known, and very difficult to reach through experimental identification. Lastly, if we turn towards dynamic models, we could no longer rely on the curved path following control law (4), since it has been designed specifically from a kinematic model.

A second possibility, more convenient with respect to our objective, is to refine kinematic model (1) in order that it can account for sliding. In aerodynamical and nautical applications, it has been proposed to take into account for wind perturbation by adding velocity terms into vessels kinematic model, see [6], [5]. Since wind effects on vessels evolution are quite similar to sliding effects on land vehicles evolution, we propose herebelow to follow the same approach.

\section{A Model derivation}

Let us again consider Ackermann's model. When sliding occurs, the ground reaction is no longer equal to the wheel action on the ground. Figure 2 displays $\vec{F}_{\text {front }}$ and $\vec{F}_{\text {rear }}$, the resultant forces perpendicular to path $\mathcal{C}$.

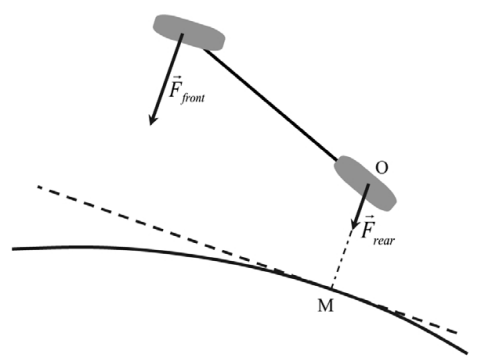

Figure 2: Forces applied on vehicle wheels

These two non equal sliding forces clearly generate:

- a resultant force which leads the vehicle to move sideways.

- a resultant torque which leads the vehicle to turn on itself.

Therefore, we propose hereafter to account for these phenomena by introducing into the vehicle model a linear lateral velocity $\dot{Y}_{p}$ and an angular one $\dot{\Theta}_{p}$. Model (1) is then refined as (the first equation has been dropped):

$$
\left\{\begin{aligned}
\dot{y} & =v \sin \tilde{\theta}+\dot{Y}_{p} \\
\dot{\tilde{\theta}} & =v\left(\frac{\tan \delta}{L}-\frac{c(s) \cos \tilde{\theta}}{1-c(s) y}\right)+\dot{\Theta}_{p}
\end{aligned}\right.
$$

\section{$B$ Model validation}

Let us first consider an academic case where sliding perturbations $\dot{Y}_{p}$ and $\dot{\Theta}_{p}$ are constant (for instance during an evolution on a field with a perfectly constant slope). In that case, it can be easily derived that the control law (4) designed under non-sliding assumption leads to asymptotic guidance errors. More precisely, from (5), we immediately obtain that:

$$
\begin{gathered}
\tilde{\theta} \stackrel{t \rightarrow \infty}{\longrightarrow}-\arcsin \left(\frac{\dot{Y}_{p}}{v}\right) \\
\frac{\tan \delta}{L} \stackrel{t \rightarrow \infty}{\longrightarrow}-\frac{\dot{\Theta}_{p}}{v}+\frac{c(s) \cos \tilde{\theta}}{1-c(s) y}
\end{gathered}
$$

Reporting (7) into (4) shows that:

$$
\frac{\cos ^{3} \tilde{\theta}}{(1-c(s) y)^{2}}(\alpha y+\beta) \stackrel{t \rightarrow \infty}{\longrightarrow}-\frac{\dot{\Theta}_{p}}{v}
$$

where:

$$
\alpha=\frac{d c(s)}{d s} \tan \tilde{\theta}+c(s) \tan \tilde{\theta}\left(K_{d}-c(s) \tan \tilde{\theta}\right)-K_{p}
$$$$
\beta=\tan \tilde{\theta}\left(c(s) \tan \tilde{\theta}-K_{d}\right)
$$

And finally, by neglecting term $y^{2}$ in (8), we prove that:

$$
y \stackrel{t \rightarrow \infty}{\longrightarrow}-\frac{\beta+\frac{\dot{\Theta}_{p}}{v \cos ^{3} \tilde{\theta}}}{\alpha-\frac{2 c(s) \dot{\Theta}_{p}}{v \cos ^{3} \tilde{\theta}}} \triangleq y_{c}
$$

Provided that $c(s)$ is constant or slowly varying, relations (9) and (6) and finally relation (7) establish that state variables $y$ and $\tilde{\theta}$ as well as control variable $\delta$ asymptotically converge to constant or slowly varying non null values. This indicates clearly that, in response to sliding occurrence, the vehicle moves crabways, which is consistent with experimental observations.

Consistency of model (5) has also been investigated through experimentation carried out with the farm tractor depicted on forthcoming Figure 9. The solid line on Figure 3 displays the evolution of the lateral deviation $y$ when automatic guidance law (4) was trying to keep the vehicle along a straight line recorded on a sloping field. Lateral deviation $y$ clearly exhibits an offset, which is not constant since the slope, in actual situation, is never perfectly constant. The dashed line on the same figure displays the evolution of $y$ simulated from control law (4) and extended model (5). Constant perturbation parameters $\dot{Y}_{p}$ and $\dot{\Theta}_{p}$ have been chosen empirically, in order that experimental and simulated asymptotic deviation roughly 
fit together $\left(\dot{Y}_{p}=-0.11 \mathrm{~m} . \mathrm{s}^{-1}\right.$ and $\left.\dot{\Theta}_{p}=0.022 \mathrm{rad} . \mathrm{s}^{-1}\right)$. It has been observed, on one hand that the asymptotic values of state variable $\tilde{\theta}$ and control variable $\delta$ simulated from model (5) also roughly fit with the experimental asymptotic values, and on the other hand that the time evolution of all variables provided by the simulation coincides with the experimental time evolution, see Figure 3. Extended model (5) appears therefore consistent.

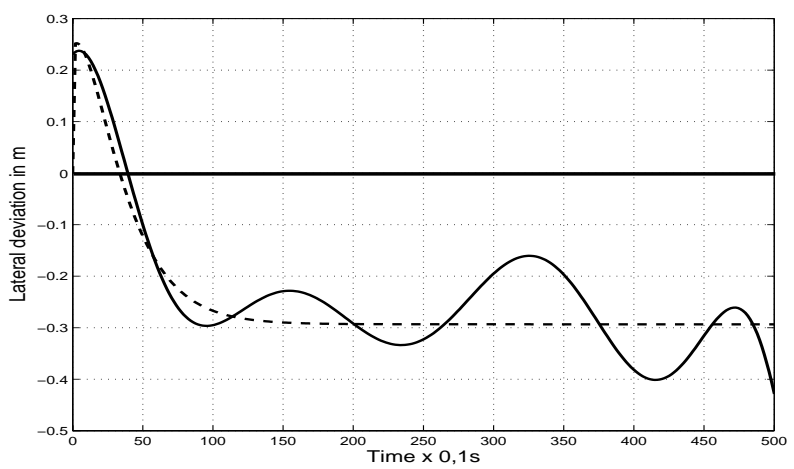

Figure 3: Comparison of lateral deviation under sliding between model and experiment

\section{GENERAL VEHICLE GUIDANCE LAW}

Since sliding effects have been incorporated into model (5) as additional terms, it might be seen quite natural to reject these perturbations by introducing into control law (4) integral correction terms. However, during field works, sliding is definitely not a static perturbation. On the contrary, its dynamic might be sometimes very fast. Therefore, integral correction terms do not appear very suitable for this application: they cannot prevent from transient large guidance error when sliding occurs. This has also been corroborated by full scale experiments.

Adaptive control can be seen as an alternative approach: for instance, a guidance system dedicated to harvesting tasks, and relying on a video camera sensor, has been proposed in [3]. A kinematic variable, namely the heading deviation, is considered as representative of vehicle sliding. Then, a correction term, computed from this variable according to an empirical relation, is incorporated into the guidance law designed under non-sliding assumption, in order to achieve sliding rejection. In this paper, a more general adaptive control framework, namely model-based techniques (see [1]), is used to deal with sliding effects. Moreover, the proposed control law still relies upon nonlinear control law (4), thus preserving all its advantages.

\section{A Adaptive control law design}

As it has been above-established, in the academic case where sliding perturbation terms $\dot{Y}_{p}$ and $\dot{\Theta}_{p}$ are constant, control law (4) leads to an asymptotic guidance error, whose value $y_{c}$ is given by (9). If $c(s)$ is also constant, this guidance error can be easily cancelled by taking into account for it inside control law (4): if the vehicle is controlled via $\delta\left(y+y_{c}, \tilde{\theta}\right)$ instead of $\delta(y, \tilde{\theta})$, computations similar to (8)-(9) establish that $y$ converges to 0 , as desired. Guidance accuracy is then preserved, despite the vehicle is still moving crabways due to sliding occurrence (since relations (6)-(7) are unchanged).

In the actual case, $\dot{Y}_{p}, \dot{\Theta}_{p}$ and $c(s)$ are not constant. However, in view of the above discussion, control law $\delta\left(y+y_{c}, \tilde{\theta}\right)$ can clearly improve guidance accuracy, once $y_{c}$ can be estimated in realtime. Such a control law is now detailed. Sliding detection and sliding correction rely upon model-based adaptive control techniques.

Sliding detection: the control law sent to the vehicle actual steering system is also used to drive the online simulation of model (1). Since this model describes the vehicle evolution in sliding absence, any difference between the simulated and the actual vehicle evolution reveals sliding occurrence. The two evolutions are compared at each time sample, see Figure 4, and disagreement in $y$ and $\tilde{\theta}$ evolution provides with the current values of the sliding parameters $\dot{Y}_{p}$ and $\dot{\Theta}_{p}$.

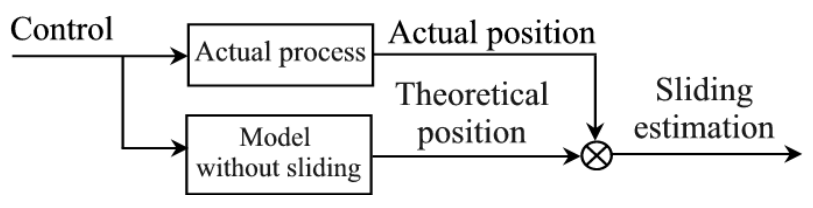

Figure 4: Sliding detection

Sliding correction: the corrective value $y_{c}$ to be introduced into the vehicle guidance law $\delta\left(y+y_{c}, \tilde{\theta}\right)$ can be derived according to a Model Reference Adaptive Control (MRAC) structure or a Internal Model Control (IMC) structure.

MRAC structure is displayed on Figure 5. Vehicle extended model $(5)$, driven by control law $\delta(y, \tilde{\theta})$ (relation (4)), and fed by the current values of sliding variables $\dot{Y}_{p}$ and $\dot{\Theta}_{p}$, is simulated in realtime. When $\dot{Y}_{p}$ and $\dot{\Theta}_{p}$ are constant, the simulated state variable $y$ converges to the desired value $y_{c}$ (see above-discussion). In the general

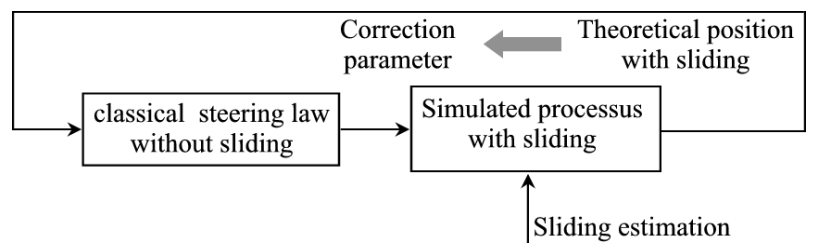

Figure 5: MRAC structure 
case, where $\dot{Y}_{p}$ and $\dot{\Theta}_{p}$ are varying, the simulated state variable $y$ is chasing, as close as possible, the optimum value of $y_{c}$ to be introduced into the adaptive guidance law $\delta\left(y+y_{c}, \tilde{\theta}\right)$.

IMC structure is depicted on Figure 6. In this approach, $y_{c}$ is directly computed from relation (9).

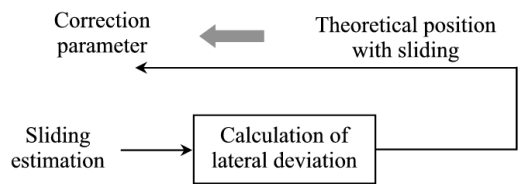

Figure 6: IMC structure

The overall adaptive control scheme is shown on Figure 7: the adaptation module is either the MRAC or the IMC structure depicted on Figure 5 or 6 . They both provide $y_{c}$ value to be used in the adaptive control law $\delta\left(y+y_{c}, \tilde{\theta}\right)$

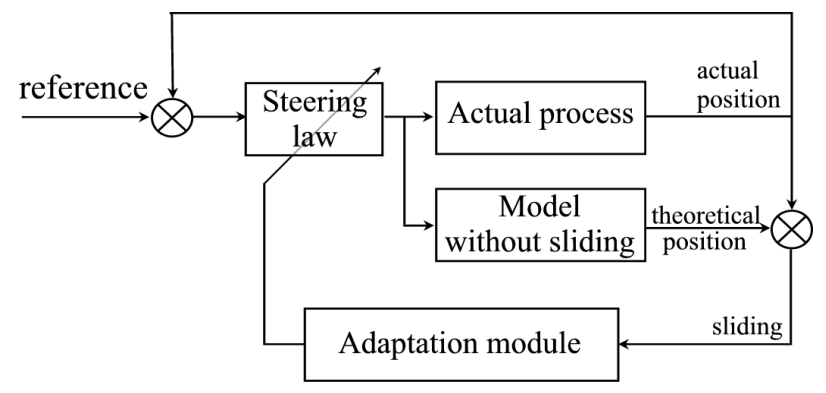

Figure 7: Overall adaptive control scheme

\section{$B$ Simulation results}

Firstly, simulation results in the academic case, where sliding parameters and reference path curvature are all constant, are displayed on Figure 8. More precisely, simulation parameters are $\dot{Y}_{p}=-0.1 \mathrm{~m} . \mathrm{s}^{-1}, \dot{\Theta}_{p}=0.03$ rad.s ${ }^{-1}, K_{p}=0.09, K_{d}=0.6$ and $c(s)=0$ (i.e. the path to be followed is a straight line). The solid line shows the evolution of the guidance error when model (5) simulation is driven via control law $\delta(y, \tilde{\theta})$, which does not take into account for sliding occurrence. The guidance error asymptotically converges to a non null value, more precisely to $48 \mathrm{~cm}$. On the contrary, when adaptive control law $\delta\left(y+y_{c}, \tilde{\theta}\right)$ is used, relying upon a MRAC structure (dash-dotted line) or upon a IMC structure (dashed line), the guidance error converges to 0 , as expected.

We can observe that the guidance error settling time is shorter when the adaptive control law relies upon the IMC structure. It is quite natural in academic cases: with IMC structure, the required value for the corrective action $y_{c}$ is immediately used in control law (see Figure 6). On

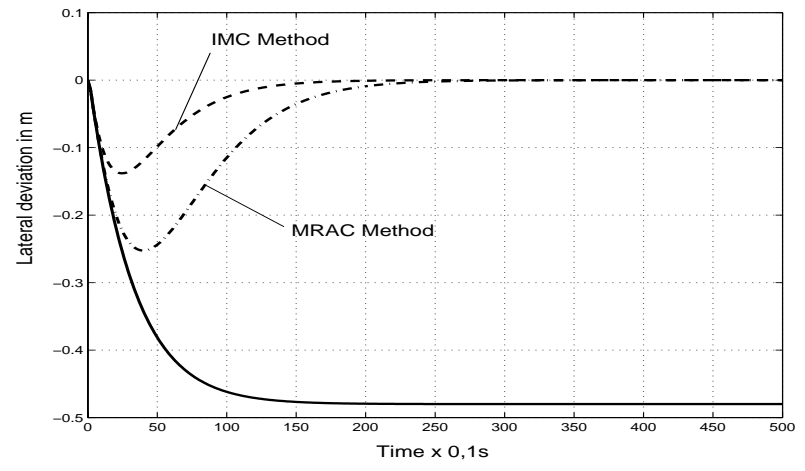

Figure 8: Simulation results when sliding parameters are constant

the contrary, with MRAC structure, this required value for $y_{c}$ is only obtained when the model (5) simulation (see Figure 5) has converged. Therefore, the settling time cannot be shorter than the system settling time (as it can be observed on Figure 8, when comparing solid line and dash-dotted line evolutions).

However, this difference in the efficiency of the two adaptation structures vanishes when considering actual experimentations. Since actual measurements are inevitably corrupted by noise, relation (9) on which relies IMC structure, cannot be used straightforwardly: all actual data have to be carefully filtered out, in order to observe a sound vehicle behaviour. The guidance error settling time is then equivalent to those obtained with MRAC structure, where model (5) simulation acts as a natural filter.

\section{EXPERIMENTAL RESULTS}

Experiments have been carried out in our laboratory farm in Montoldre, France. The farm tractor is depicted on Figure 9. The kinematic GPS receiver is a Thales Navigation dual frequency "Aquarius 5002" unit, providing position and velocity measurements with a $2 \mathrm{~cm}$ accuracy, at a $10 \mathrm{~Hz}$ sampling frequency. The GPS antenna has been located on the tractor cabin, straight up the point $O$ (see Figure 1), in order that the GPS receiver provides directly the location of that point. The adaptive control laws $\delta\left(y+y_{c}, \tilde{\theta}\right)$ has been implemented in high level language $(\mathrm{C}++)$ on a Pentium based computer.

Land vehicles undergo sliding effects, either when they enter into a curve on a wet land, or when they move on a sloping field. The experimental results reported below address the first situation. Experimentations on sloping fields have however been carried out, and display the same positive behaviour.

The path to be followed is depicted on Figure 10. It con- 


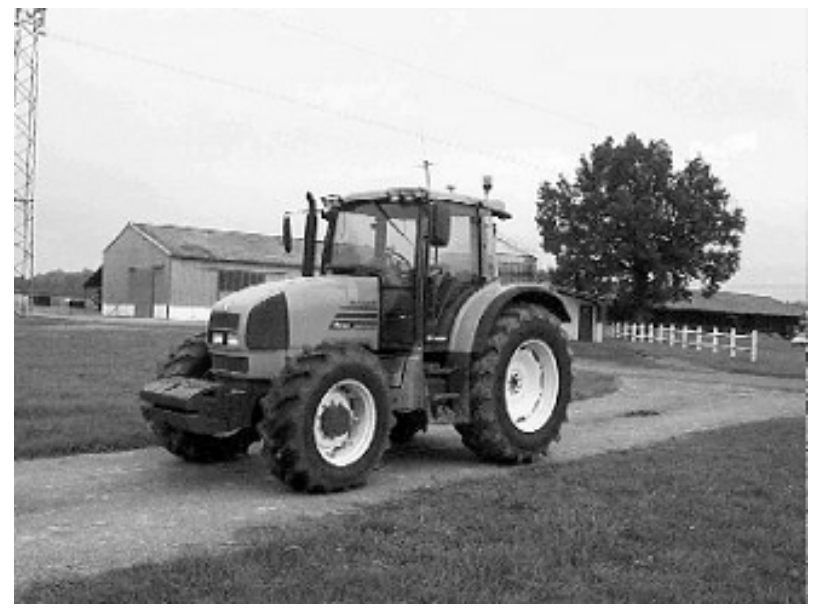

Figure 9: Experimental vehicle

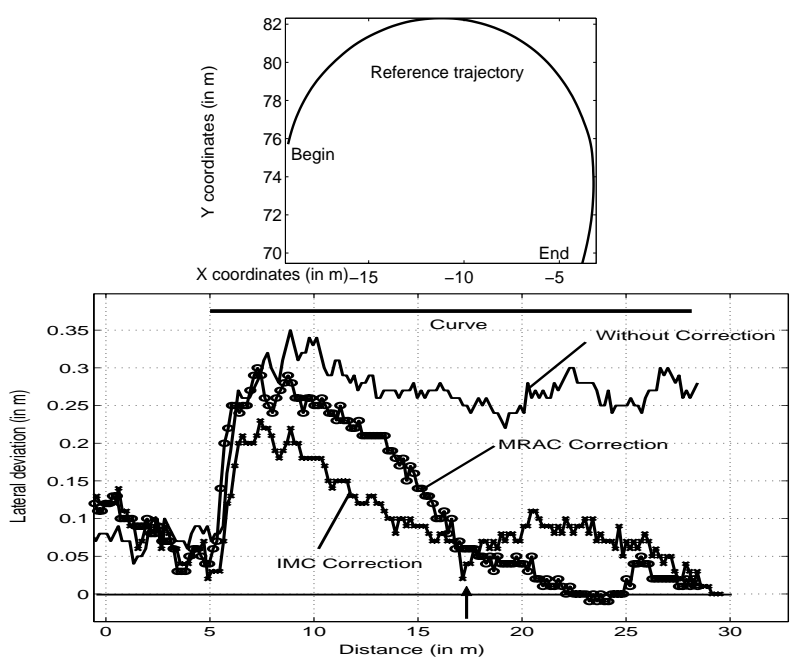

Figure 10: Experiment on a curve

sists mainly in a constant curve on a gravelled ground. Control law (4), which encloses no sliding corrective terms, leads to an almost constant lateral deviation (reflecting constant sliding conditions). On the contrary, both adaptive structures ensure that the lateral deviation returns to 0 . As expected from previous simulations (see Figure 8), IMC structure exhibits on Figure 10 a higher convergence rate than the MRAC one. However, it is more sensitive to noise and perturbations: when the tractor has covered $17 \mathrm{~m}$ (see the arrow on Figure 10), it has run into a hole. Sliding correction via IMC structure is altered, whereas MRAC one still shows a satisfactory behaviour.

\section{CONCLUSION}

In this paper, model-based adaptive techniques have been used in order to take into account for sliding effects in ve- hicle automatic guidance control laws. More precisely, MRAC and IMC structures have been investigated to refine a nonlinear control law, designed from chained systems theory, and thus propagating all linear systems features to vehicle nonlinear model. Theoretical results have been validated by experimentations carried out with a farm tractor, using a kinematic GPS as unique localization sensor.

Experimental results are quite satisfactory on almost regular ground. However, on uneven grounds, the GPS antenna located on the top of tractor cabin undergoes shocks, which are wrongly understood as sliding by the adaptive control laws. Specific filtering operations have then to be considered. We are also working on the integration of a low-cost GPS attitude sensor, which could provide us with cabin vibrations.

References

[1] Borne P., Dauphin-Tanguy G., Rotella F. and Zambettakis I. Commande et optimisation des processus. Series : Méthodes et pratiques de l'ingénieur, TECHNIP Editions, Paris (France) 1990. [2] Cordesses L., Thuilot B., Martinet P. and Cariou C. Curved path following of a farm tractor using a CP-DGPS. In Proc. of the $6^{\text {th }}$ Intern. Symposium on Robot Control (Syroco), pp 13-18, Vienna (Austria), 2000.

[3] Debain C., Chateau T., Berducat M., Bonton P., and Martinet P. A guidance system for agricultural vehicles In Computers an Electronics in Agriculture, Special issue navigation Agricultural Field Machinery, 25(1-2):29-51, January 2000.

[4] Ellouze M. and d'Andréa-Novel B. Control of unicycle-type robots in the presence of sliding effects with only absolute longitudinal and yaw velocities measurement. In European Journal of Control, 6:567-584, 2000.

[5] Holzhüter T. and Schultze R. Operating experience with a high-precision track controller for commercial ships. In Control Engineering Practice 4(3):343-350, 1996.

[6] Loeb H. Engin nautique de surface robotisé : synthèse de lois de commande et GPS différentiel $\mathrm{PhD}$ Thesis, University of Bordeaux I (France), July 1996.

[7] Nagasaka Y., Otani R., Shigeta K. and Taniwaki K. Automated operation in paddy fields with a fiber optic gyro sensor and GPS. In Proc. of the Intern. Workshop on Robotics and Automated Machinery for Bio-Productions (Bio-Robotics), pp 21-26, Valencia (Spain) September 1997.

[8] O'Connor M., Elkaim G., Bell T. and Parkinson B. Automatic steering of a farm vehicle using GPS. In Proc. of the $3^{\text {rd }}$ Intern. Conf. on Precision Agriculture, Minneapolis (USA), pp 767777, June 1996.

[9] Ried J. and Niebuhr D. Driverless tractors. In Ressource 8(9):7-8, September 2001.

[10] Samson C. Control of chained systems. Application to path following and time-varying point-stabilization of mobile robots. In IEEE Trans. on Automatic Control 40(1):64-77, January 1995.

[11] Thuilot B., Cariou C., Martinet P. and Berducat M.. Automatic guidance of a farm tractor relying on a single CP-DGPS. In Autonomous robots 13(1):53-71, July 2002.

[12] Thuilot B. Contribution à la modélisation et à la commande de robots mobiles à roues. PhD Thesis, Ecole des Mines de Paris (France), December 1995.

[13] The Zodiac. Theory of robot control. Canudas de Wit C., Siciliano B. and Bastin G. eds, Springer Verlag, Berlin (Germany) 1996. 